SECTION 31. Economic research, finance, innovation.

Anatoly Aleksandrovich Naumov

Docent, Candidate of Technical Sciences, Center of Applied Mathematical Research, Novosibirsk, Russia,

E-mail: a_a_naumov@mail.ru

\title{
ANALYSIS OF RECURRENT SHEMES AND DIFFERENTIAL EQUATIONS FOR FINANCE ANALYSIS PROBLEMS
}

\begin{abstract}
The paper discusses recurrent schemes and differential equations for finance analysis problems.

Keywords: Finance analysis, recurrent schemes, differential equations, finance flows, models.

\section{УДК 336.761: 336.748}

\section{СРАВНИТЕЛЬНЫЙ АНАЛИЗ ИСПОЛЬЗОВАНИЯ РЕКУРРЕНТНЫХ СХЕМ И ДИФФЕРЕНЦИАЛЬНЫХ УРАВНЕНИЙ В ЗАДАЧАХ ФИНАНСОВОГО АНАЛИЗА}

\begin{abstract}
Аннотация: $B$ работе приведены результаты анализа разностных дифференциальных уравнений и генерирующих соотношений при решении задач анализа финансовых потоков. Показано преимущество генерируюших соотношений.

Ключевые слова: Финансовый анализ, рекуррентные схемы, дифференциальные уравнения, финансовые потоки, модели.
\end{abstract}

Работа появилась в связи с публикацией [1], в которой предлагается использовать дифференциальные уравнения в разностной форме для получения некоторых характеристик финансовых потоков.

Постановка задачи такова. Имеется некоторый финансовый поток. Для него существует генерирующее соотношение (выражение). Такое соотношение позволяет найти значения элементов потока в любой из моментов времени на интервале существования финансового потока. Будем считать, без умаления общности и в соответствии с рассмотренными моделями потоков в работе [1], финансовые потоки дискретными во времени. В качестве примеров генерирующих соотношений можно привести следующие:

1) генерирующее соотношение рекуррентного типа, когда каждый из элементов финансового потока в некоторый момент времени, находится в виде некоторой функции от предыдущих (предыдущего) значений потока и некоторых параметров;

2) генерирующее соотношение задается в виде некоторого алгоритма, с помощью которого можно найти значение финансового потока в любой момент времени.

В качестве параметров генерирующих соотношений могут выступать процентные ставки (заемного процента, внешнего использования и др.), моменты времени активностей элементов финансовых потоков (времена пополнения потоков новыми финансовыми средствами, времена изъятия денежных средств и т.д.), непосредственно значения финансовых средств для пополнения и изъятия из потоков, величины интервалов времени между точками активностей и т.д. Как правило, параметры генерирующих соотношений известны заранее для каждого момента временили или могут быть найдены с помощью некоторого алгоритма (алгоритма генерирования параметров). 
Требуется на основе генерирующих соотношений финансового потока определить некоторый набор его характеристик. В качестве таких характеристик может выступать, например, любой из показателей эффективности финансовых потоков. В простейших случаях - это значение обобщенного (суммарного, итогового) потока в некоторый момент времени.

Задача решается достаточно просто. Для этого следует воспользоваться генерирующим соотношением и последовательно во времени (если задана, например, рекуррентная формула) найти требуемые характеристики [3].

В работе [1] предлагается решать задачу нахождения характеристик финансовых потоков с использованием дифференциальных уравнений, построенных на основе генерирующих соотношений. Можно сказать, что авторы предлагают воспользоваться неким «обходным маневром» с использованием аппарата дифференциальных уравнений для решения этой задачи. Как поется в известной песне: «Нормальные герои всегда идут в обход». Чтобы такой прием привлек внимание исследователей и за него ухватились бы практики, необходимо выполнение нескольких условий:

1) необходимо, чтобы проведение расчетов через дифференциальные уравнения давал бы некоторые преимущества по сравнению с непосредственным использованием генерирующих соотношений; например, такими преимуществами могут быть: большая точность расчета характеристик потоков, меньшие затраты времени на их вычисление и т.Д.;

2) необходимо, чтобы был возможен переход от генерирующих соотношений к дифференциальным уравнениям;

3) необходимо, чтобы полученные дифференциальные уравнения имели аналитическое решение.

Заметим, что, к сожалению, в общем случае прохождение через «обходной маневр» не всегда возможен. Кроме этого, при одинаковых получаемых выражениях характеристик для схем с использованием генерирующих выражений и дифференциальных уравнений нет преимуществ ни в точности, ни в затратах временного ресурса на нахождение таких характеристик. Возникает уместный вопрос: зачем прибегать к расчетной схеме (с использованием дифференциальных уравнений), преимущества которой неочевидны? Рассмотрим некоторые особенности генерирующих соотношений, которые не позволяют в принципе воспользоваться «обходным маневром».

Случай зависимости параметров генерирующих соотношений от значений элементов финансового потока. Иногда на практике приходится иметь дело с такими особенностями элементов финансовых потоков, когда проявляется зависимость параметров от значений элементов этих потоков. Например, банковская ставка может зависеть от размеров депозитных средств. Ставка заемного процента может зависеть от размеров займа и т.д. Во всех таких случаях переход к дифференциальным уравнениям не позволит найти их решение в аналитическом виде (точно).

Случай алгоритмически вычислимых характеристик финансовых потоков. В этом случае нет возможности получить характеристики потока в виде некоторой формулы (в аналитическом виде) [6-10]. Так происходит, например, при оценивании эффективности проектов, когда используются схемы на основе анализа будущего приведенного дохода. При этом на каждом из шагов такого анализа принимаются решения о возможности (или невозможности) вывода финансовых средств за пределы проекта (например, во внешние для данного проекта проекты), принимаются решения о погашении кредитов и т.д. В таких случаях невозможно (по сравнению с классическими подходами) представить характеристику (показатель эффективности) в виде некоторой формулы (выражения, компактно, аналитически). Заметим, что в таком представлении нет большой необходимости. Главное, чтобы такая характеристика была бы вычислимой в принципе и для нее существовал бы алгоритм, который можно было бы реализовать с использованием 
вычислительной техники [6; 7]. Назовем ситуацию, когда не существует формулы для расчета характеристики финансовых потоков, а существует некоторый алгоритм, алгоритмически вычислимой, а соответствующие этому потоку характеристики алгоритмически вычислимыми характеристиками (ABX). Очевидно, для $\mathrm{ABX}$ использование в качестве схемы для их расчетов дифференциальных уравнений (использование «обходного маневра») представляется невозможным.

Расчетные схемы финансового анализа в пакете программ MatLab. B пакете программ MatLab можно воспользоваться функциями финансового анализа, построенными на схеме прямого использования генерирующих соотношений.

Выводы. Использование аппарата дифференциальных уравнений в качестве «обходного маневра» для решения задач оценивания характеристик финансовых потоков представляется неэффективным. В некоторых случаях (причем тех, которые наиболее близки к практическому использованию) такое использование невозможно (нереализуемо). При выборе схем расчета характеристик финансовых потоков, на наш взгляд, следует отдавать предпочтение методам, использующих генерирующие соотношения напрямую, в том числе - рекуррентным схемам. Генерирующие соотношения позволяют существенно расширить класс задач оценивания характеристик финансовых потоков, включая те задачи, в которых используются АВХ [6-10].

\section{References:}

1. Кириллов Ю.В., Назимко Е.Н. Использование разностных уравнений для решения задач финансовой математики// Финансовая аналитика: проблемы и решения, 2014, № 20 (206), C. 58-64.

2. Разностные уравнения и их приложения// А.Н. Шарковский и др. - Киев: Наукова думка, 1986. - 280 с.

3. Шарп У., Александер Г., Бейли Дж. Инвестиции. - М.: ИНФРА-М, 2001. - 1028 с.

4. Arnold V.I. Ordinary Differential Equations// Second printing of the 1992 ed. - SpringerVerlag, Berlin, 2006.

5. Fusai G., Sanfelici S., Tagliani A. Practical Problems in the Numerical Solution of PDE's in Finance// Rend. Studi Econ. Quant., 2001, pp. 105-132.

6. Наумов А.А., Ходусов Н.В. Управление портфельными инвестициями. Модели и алгоритмы. - Новосибирск: ОФСЕТ, 2005. - 298 с.

7. Наумов А.А., Шубин Д.А. К задаче построения эффективного портфеля проектов// Сборник научных трудов НГТУ, 2006, № 3 (45), С. 145-148.

8. Наумов А.А., Бах С.А. Бизнес-процессы. Синтез, анализ, моделирование и оптимизация. - Новосибирск: ОФСЕТ, 2007. - 307 с.

9. Наумов А.А. Теоретические и прикладные вопросы моделирования бизнес-процессов. Модели, алгоритмы, программы. - LAP LAMBERT Academic Publishing, 2012. - 464 с.

10. Наумов А.А. Методы анализа и синтеза инвестиционных проектов. Эффективность, риски, управление. - LAP LAMBERT Academic Publishing, 2013. - 356 с. 